\title{
Interleukin-11 Levels in Healthy and Thrombocytopenic Neonates
}

\author{
MARY P. McCLOY, IRENE A.G. ROBERTS, LUCY J. HOWARTH, TIMOTHY L. WATTS, AND \\ NEIL A. MURRAY \\ Departments of Paediatrics and Neonatal Medicine [L.J.H., T.L.W., N.A.M.] and Haematology [M.P.M., \\ I.A.G.R.], Faculty of Medicine, Imperial College, Hammersmith Hospital Campus, London W12 ONN, U.K.
}

\begin{abstract}
Thrombocytopenia is common in sick neonates, and affected neonates have adverse outcomes compared with those without thrombocytopenia. As impaired platelet production underlies many neonatal thrombocytopenias, affected neonates are potential candidates for hemopoietic growth factor therapy. Although recombinant human (rh) thrombopoietin remains under therapeutic development, rhIL-11, which stimulates megakaryocytopoiesis and increases platelet counts after chemotherapy, is already licensed for clinical use. However, nothing is known about IL-11 in neonates. We therefore measured plasma IL-11 by ELISA in healthy term neonates, stable preterm neonates with or without thrombocytopenia, and preterm neonates with sepsis or necrotizing enterocolitis (NEC) with or without thrombocytopenia. At birth IL-11 was undetectable $(<10 \mathrm{pg} / \mathrm{mL})$ in healthy term neonates $(n=20)$ and 27 of $31(87 \%)$ stable preterm neonates. Three stable preterm neonates with detectable IL-11 (mean \pm SD, $11.3 \pm 0.4 \mathrm{pg} / \mathrm{mL}$; median, $11.6 \mathrm{pg} / \mathrm{mL}$ ) suffered chorioamnionitis, the remaining neonate (IL-11, $14 \mathrm{pg} / \mathrm{mL}$ ) being one of nine with early onset thrombocytopenia (present by $<72 \mathrm{~h}$ of age). IL-11 was also measured in 58 preterm neonates with suspected sepsis or NEC. In 25 of 58, sepsis or NEC was unconfirmed and IL-11 was undetectable. By contrast, 14 of 33 with proven sepsis or NEC had elevated IL-11 (median, $14.9 \mathrm{pg} / \mathrm{mL}$; range, 11.2-
\end{abstract}

\section{ABSTRACT}

$92.2 \mathrm{pg} / \mathrm{mL})$. Of these 33 neonates, 19 developed thrombocytopenia: nine of 19 (47\%) had detectable IL-11 and 10 of $19(53 \%)$ did not $(p>0.05)$. Although its role in platelet production in neonates remains unclear, these data suggest that IL-11 is involved in the endogenous cytokine response to sepsis or NEC in preterm neonates. Further studies of IL-11 in neonates are warranted to assess its role both in platelet production and in mediation of the endogenous inflammatory response. (Pediatr Res 51: 756-760, 2002)

Tep, thrombocytopenia

rhIL-11, recombinant human IL-11

HGF, hemopoietic growth factor

NEC, necrotizing enterocolitis

PET, preeclampsia

Tpo, thrombopoietin

GA, gestational age

NICU, neonatal intensive care unit

PROM, prolonged rupture of membranes

IUGR, intrauterine growth restriction

CRP, C-reactive protein
Thrombocytopenia (platelets $<150 \times 10^{9} / \mathrm{L}$ ) occurs in $25 \%$ of neonates admitted to NICU $(1,2)$. Affected neonates are reported to be at increased risk of intracranial hemorrhage (1, 3-7), mortality (1-3), and long-term morbidity (5). As a result, many thrombocytopenic neonates receive repeated platelet transfusions $(8,9)$ although there is little evidence that this improves outcome (10). However, it does increase their risk of transfusion-related complications, particularly infection (11).

Received November 29, 2000; accepted January 11, 2002.

Correspondence and reprint requests: Neil A. Murray, M.D., Department of Paediatrics and Neonatal Medicine, Hammersmith Hospital, Du Cane Road, London W12 OHS, U.K.; e-mail: neil.murray@ic.ac.uk

Supported by funding from the following charitable organizations: Action Research, Children Nationwide, and Sir Halley Stewart Trust.

DOI: 10.1023/01.PDR.0000015102.98439.6C
Impaired megakaryocytopoiesis and platelet production is increasingly recognized as a major contributory cause to many neonatal thrombocytopenias $(2,12,13)$. This suggests that HGFs, which stimulate megakaryocytopoiesis, may offer an alternative approach to platelet transfusion. Tpo is the main HGF regulator of platelet production in neonates (13-16), and endogenous Tpo levels during neonatal thrombocytopenia may be suboptimal $(13,15)$, making Tpo an attractive HGF option for thrombocytopenic neonates. However, rhTpo is not currently available for therapeutic use. In contrast the thrombopoietic cytokine rhIL-11 is already in clinical use. Trials show that rhIL-11 stimulates megakaryocytopoiesis both in adults and children thereby increasing platelet counts in thrombocytopenic patients (17-19). However, little is known about endogenous IL-11 in neonates $(20,21)$, and rhIL-11 has not been used for thrombocytopenic neonates. 
To investigate the role of IL-11 in neonatal thrombopoiesis, we measured plasma IL-11 in healthy term and stable preterm neonates and in preterm neonates at risk of developing two different presentations of neonatal thrombocytopenia (12): 1) early onset thrombocytopenia $(<72 \mathrm{~h})$ associated with maternal PET or fetal IUGR; and 2) late-onset thrombocytopenia $(>72 \mathrm{~h})$ associated with late-onset sepsis and NEC.

\section{METHODS}

\section{Patients and Samples}

The study was performed at the Hammersmith and Queen Charlotte's Hospitals, London, and approved by the local ethics committee. After obtaining informed parental consent, all blood samples were collected into sample tubes containing only preservative-free heparin $(10 \mathrm{U} / \mathrm{mL})$ and kept at $4^{\circ} \mathrm{C}$. Plasma was obtained from blood samples by centrifugation at $500 \times \mathrm{g}$ for $10 \mathrm{~min}$ within $1 \mathrm{~h}$ of collection.

Term neonates. Cord blood was obtained at birth from 20 term neonates (GA range, 37-41 wk; 11 boys, nine girls). All of these neonates were born after uncomplicated pregnancies (i.e. no history of maternal PET, diabetes, thrombocytopenia, pyrexia during labor, or drug therapy), and none of the neonates developed significant neonatal complications. Blood was collected immediately after delivery of the placenta by aspiration from placental vessels using a 21-gauge needle connected to a syringe.

Preterm neonates studied at birth. Cord blood was obtained in a total of 31 preterm neonates (GA range, 24-31 wk; 17 boys, 14 girls) and collected by aspiration from placental vessels using a 21 -gauge needle attached to a syringe. Fifteen of the 31 neonates were exposed to maternal PET or fetal IUGR, of which nine $(60 \%)$ developed early onset thrombocytopenia (platelets $<150 \times 10^{9} / \mathrm{L}$ by $72 \mathrm{~h}$ ). The remaining neonates maintained normal platelet counts during the first $72 \mathrm{~h}$. There were no significant differences in GA, severity of initial neonatal complications, and maternal drug exposure among these stable preterm neonates whether or not they had been exposed to maternal PET or suffered IUGR or early thrombocytopenia. Platelet counts were measured as part of the routine full blood count on a Sysmex SE-9000 analyzer (Sysmex UK Ltd, Milton Keynes, U.K.) and confirmed by daily examination of blood films on all NICU patients (I.A.G.R.).

Preterm neonates studied at $>72 \mathrm{~h}$ of age. Fifty-eight preterm neonates (GA range, 24-36 wk; 38 boys, 20 girls) were studied after $72 \mathrm{~h}$. Peripheral blood samples were obtained at the time of clinical sampling (using 23- to 25-gauge needles) after clinical deterioration suggesting episodes of sepsis or NEC. A diagnosis of sepsis or NEC (see below for definitions) was confirmed in 33 of 58 neonates; the remaining 25 neonates whose eventual clinical course and laboratory findings did not support a diagnosis of sepsis or NEC formed the late healthy controls (all 25 maintained platelet counts $>150 \times 10^{9} / \mathrm{L}$ ). Sepsis occurred in 22 of 33 and NEC in 11 of 33 neonates of whom $15(68 \%)$ and four $(36 \%)$ developed thrombocytopenia, respectively.

Sepsis was defined as a positive culture from blood, cerebrospinal fluid, urine, or endotracheal secretions plus one further laboratory indicator of infection: elevated CRP, chest radiograph suggesting consolidation, left shift or toxic granulation of neutrophils, or a new episode of thrombocytopenia; or in the absence of a positive culture, a clinical deterioration suggestive of sepsis in combination with two of the above indicators. NEC was defined as pneumatosis intestinalis on abdominal x-ray or magnetic resonance imaging in combination with clinical symptoms and signs of the disease. If neonates had positive evidence of both sepsis and NEC they were assigned to the NEC group.

\section{IL-11 ELISA}

Plasma samples were stored at $-20^{\circ} \mathrm{C}$ until analyzed. IL-11 levels were measured using a commercially available ELISA kit (Quantikine Human IL-11 ELISA; R\&D Systems Europe, Abingdon, Oxford, U.K.), with a lower limit of detection of 10 $\mathrm{pg} / \mathrm{mL}$.

\section{Statistics}

Comparisons among groups were performed using $\chi^{2}$ analysis; $p<0.05$ was considered significant.

\section{RESULTS}

Healthy term neonates at birth. Plasma IL-11 was undetectable $(<10 \mathrm{pg} / \mathrm{mL})$ at birth in all 20 term neonates studied (Fig. 1A).

Preterm neonates studied at birth. Plasma IL-11 at birth was $<10 \mathrm{pg} / \mathrm{mL}$ in the majority of preterm neonates studied ( 27 of $31 ; 87 \%$ ). Of the 16 neonates in this group who were not exposed to either maternal PET or suffered IUGR, three had detectable plasma IL-11 (Fig. $1 A$ ), all of them at a low level $(10.6,11.6$, and $11.8 \mathrm{pg} / \mathrm{mL})$. All three were born after pregnancies complicated by PROM, and their placental histology showed evidence of severe chorioamnionitis, although none had positive blood cultures at birth or developed early onset thrombocytopenia. Of the remaining 15 neonates who were all either exposed to maternal PET or suffered IUGR (nine of whom also developed early thrombocytopenia), only one had detectable plasma IL-11 at birth $(14 \mathrm{pg} / \mathrm{mL}$; Fig. $1 A)$. This neonate had severe idiopathic IUGR (birth weight 2 SD less than the third percentile) and was also significantly thrombocytopenic (platelet count, $36 \times 10^{9} / \mathrm{L}$ ).

Preterm neonates with suspected sepsis or NEC. Fifty-eight neonates were screened for suspected sepsis or NEC at $>72 \mathrm{~h}$ and had concurrent plasma IL-11 levels measured. Their clinical details are shown in Table 1. In the 25 neonates in whom sepsis or NEC was unconfirmed and who maintained normal platelet counts, plasma IL-11 was undetectable (Fig. 1B). In contrast in the 22 neonates with sepsis and 11 with NEC, plasma IL-11 was detectable in 10 of $22(45 \%$; range, $11.2-$ $41.5 \mathrm{pg} / \mathrm{mL}$; Fig. $1 B)$; and four of 11 (36\%; range, 11.6-92.2 $\mathrm{pg} / \mathrm{mL}$; Fig. $1 B)$. The difference between plasma IL-11 levels in the group of neonates with sepsis or NEC $(n=33)$ compared with the group of neonates without these conditions $(n=$ 25 ) was highly significant $(p<0.001)$. However, there was no significant relationship between plasma IL-11 and clinical severity of sepsis or NEC as reflected by CRP on the day of 
(A)

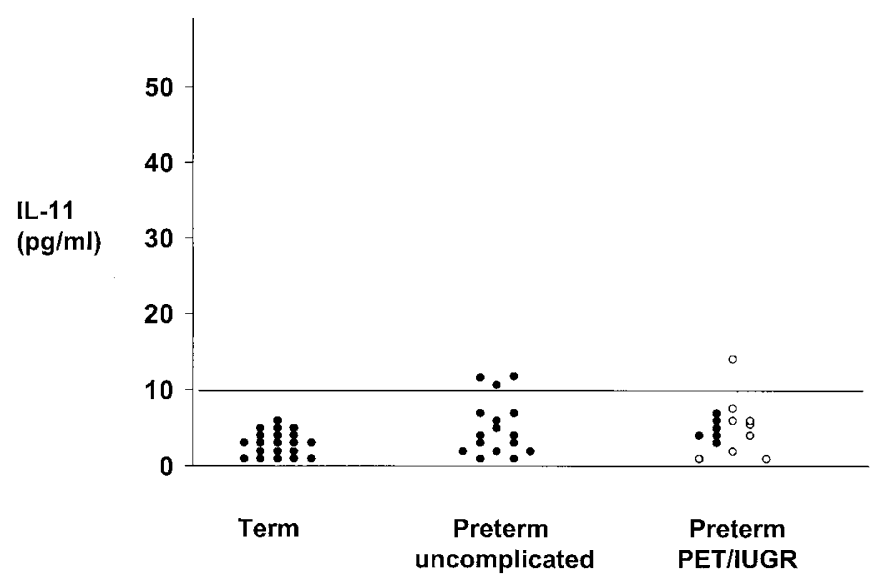

(B)

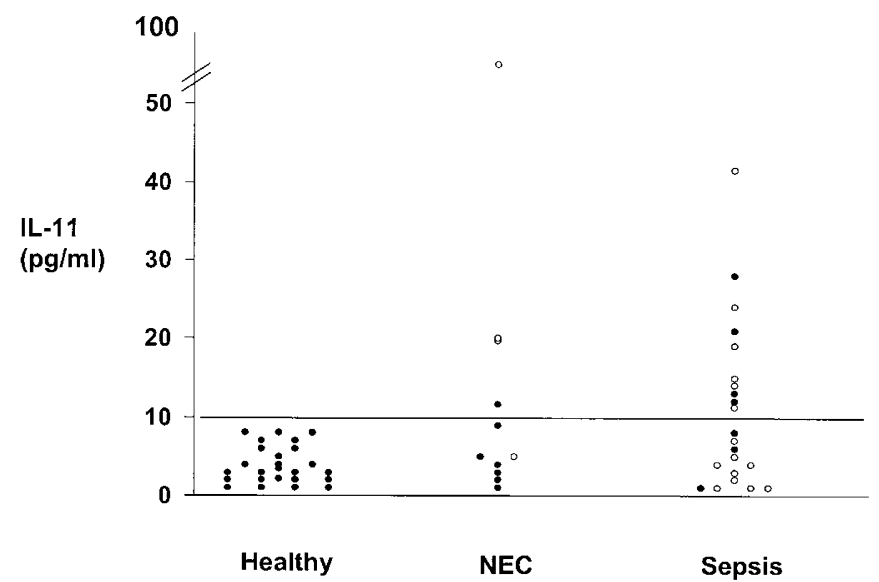

Figure 1. Plasma IL-11 levels in 109 term and preterm neonates. $A$, at birth $(n=51)$ plasma IL-11 was detectable $(>10 \mathrm{pg} / \mathrm{mL}$, solid line) in none of the 20 term neonates, in three of the 16 preterm neonates with no history of placental insufficiency (maternal PET/IUGR) and in one of the 15 neonates with a history of placental insufficiency (severe IUGR). neonates with normal platelet counts; $O$, thrombocytopenic neonates (platelets $<150 \times$ $\left.10^{9} / \mathrm{L}\right) . B$, at $>72 \mathrm{~h}$ of life $(n=58)$ plasma IL-11 levels were significantly higher $(p<0.001)$ in the group of neonates with sepsis or NEC $(n=33)$ compared with the group of neonates without these conditions $(n=25)$.

sampling or peak CRP, or survival (Table 1). Nineteen of the 33 neonates with sepsis or NEC developed thrombocytopenia. Of these, nine of 19 (47\%) had detectable plasma IL-11, and 10 of $19(53 \%)$ did not $(p>0.05)$. In addition, there was no significant correlation between plasma IL-11 and platelet count in the preterm neonates studied as a single group (Fig. 2).

\section{DISCUSSION}

Recent evidence suggests that hyporegenerative thrombocytopenia is common in sick neonates. Clinical trials show that rhIL-11 stimulates human megakaryocytopoiesis (17-19), making rhIL-11 a potential therapy for thrombocytopenic neonates. However, there are few data about IL-11 and its role in thrombopoiesis in neonates. To investigate this we measured plasma IL-11 levels at birth in healthy term neonates and at birth and during the neonatal period in stable preterm neonates and preterm neonates at high risk of developing thrombocytopenia (12).

We found that plasma IL-11 levels were undetectable at birth in healthy term neonates and the vast majority of stable preterm neonates. As the level of sensitivity of the IL-11 ELISA was $10 \mathrm{pg} / \mathrm{mL}$, this indicates that the normal, physiologic level of circulating IL-11 at birth is $<10 \mathrm{pg} / \mathrm{mL}$. Only four of the 31 preterm neonates tested at birth had detectable IL-11 (three suffered PROM or chorioamnionitis and one had severe idiopathic fetal IUGR and thrombocytopenia). By contrast, almost half of the 33 neonates with sepsis or NEC had elevated plasma IL-11 $(>10 \mathrm{pg} / \mathrm{mL})$. However, despite the known effects of IL-11 on megakaryocytopoiesis in vitro in adults and in vivo in adults and children with chemotherapyinduced thrombocytopenia (17-19), we found no correlation between plasma IL-11 levels and platelet count in the neonates we studied. The majority of neonates with thrombocytopenia did not have elevated plasma IL-11, and elevated IL-11 levels were found equally in neonates with and without thrombocytopenia.

Although comparison of data among different age groups has to be interpreted with caution, our data are consistent with those of Cremer et al. (22), who measured IL-11 levels in thrombocytopenic children with aplastic anemia and a variety of inherited platelet disorders and found detectable IL-11 in only $27 \%$ of thrombocytopenic children. However, by contrast, our data differ from those of Chang et al. (23), who found a close reciprocal relationship between plasma IL-11 levels and platelet count in thrombocytopenic children undergoing chemotherapy.

Together these data suggest that IL-11 plays a role in platelet production that is different from that of Tpo. For Tpo there is now good evidence that plasma levels consistently increase as megakaryocytopoiesis and platelet production decrease (24, $25)$. Given that IL-11 clearly stimulates megakaryocytopoiesis in vitro and in vivo (at least in adults and children), the absence of a correlation between plasma IL-11 and platelet count in our neonatal patients could be explained in a number of ways. First, plasma IL-11 levels may increase only in the setting of severe thrombocytopenia. Our data would generally be consistent with this: plasma IL-11 was not increased in the neonates with early thrombocytopenia secondary to maternal PET or IUGR if the thrombocytopenia was modest $\left(50-150 \times 10^{9} / \mathrm{L}\right)$. However, IL-11 was increased in the neonate with severe thrombocytopenia secondary to IUGR and in slightly less than half of the neonates with significant thrombocytopenia secondary to sepsis or NEC. Nevertheless many neonates with significant thrombocytopenia did not have detectable plasma IL11. Second, although the contribution of plasma IL-11 to platelet production might be modest, under physiologic conditions, locally produced IL-11 (not detectable in the systemic circulation), either alone or in synergy with other megakaryocytopoietic cytokines such as IL-6 and stem cell factor, may play a significant role in platelet production. Such a model would be consistent with the observed effects of IL-11 in vitro in which it seems to exert its effects predominantly on more 
Table 1. Clinical details of 58 preterm babies presenting after the age of 72 hours with suspected sepsis or NEC

\begin{tabular}{|c|c|c|c|c|}
\hline & $\begin{array}{c}\text { All babies } \\
n=58\end{array}$ & $\begin{array}{c}\mathrm{IL}-11>10 \\
\mathrm{pg} / \mathrm{ml} \\
n=14\end{array}$ & $\begin{array}{c}\mathrm{IL}-11<10 \\
\mathrm{pg} / \mathrm{ml} \\
n=44\end{array}$ & $p$ values \\
\hline Gestational age (weeks) & $\begin{array}{c}27.8 \pm 0.35 \\
23-34\end{array}$ & $\begin{array}{c}26.9 \pm 0.59 \\
23-30\end{array}$ & $\begin{array}{c}28.1 \pm 0.41 \\
23-34\end{array}$ & 0.85 \\
\hline Birth weight $(\mathrm{kg})$ & $\begin{array}{c}1.04 \pm 0.06 \\
0.65-2.98\end{array}$ & $\begin{array}{c}0.88 \pm 0.07 \\
0.65-1.2\end{array}$ & $\begin{array}{c}1.09 \pm 0.07 \\
0.5-2.98\end{array}$ & 0.83 \\
\hline Gender (male:female) & $38: 20$ & $9: 5$ & $29: 15$ & 0.91 \\
\hline Mortality & $4(7 \%)$ & $2(14 \%)$ & $2(4 \%)$ & $<0.02$ \\
\hline \multicolumn{5}{|l|}{ CRP } \\
\hline Peak & $67.4 \pm 9.5$ & $68.7 \pm 18.9$ & $67 \pm 11.2$ & 0.98 \\
\hline Day of sampling & $51.9 \pm 9.4$ & $54.2 \pm 18.6$ & $51 \pm 11$ & 0.99 \\
\hline
\end{tabular}

Values are expressed as the mean \pm SEM, range.

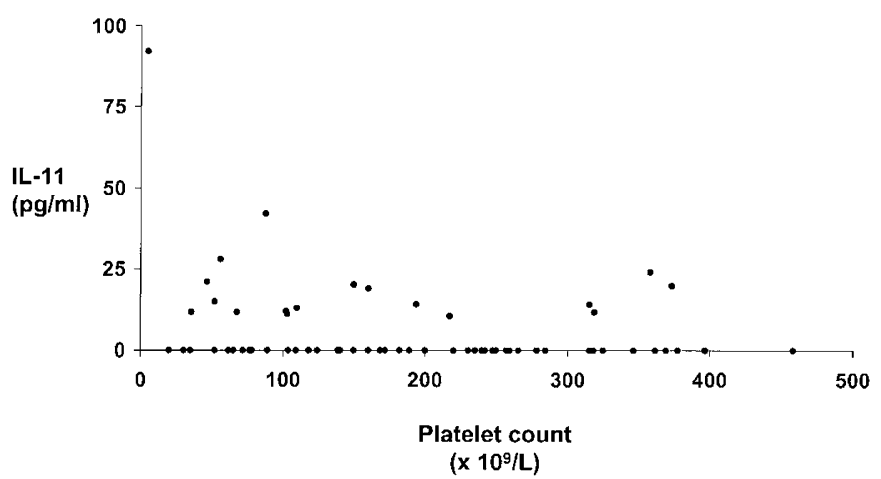

Figure 2. The relationship between plasma IL-11 and platelet count in 89 preterm neonates.

mature cells of the megakaryocyte lineage and to have a greater effect in the presence of stem cell factor and IL-6 (26). Third, we were only able to obtain a single sample for IL-11 measurement and, therefore, may have missed transiently elevated levels in some neonates. Finally, it may be that IL-11 does not play any significant role in neonatal megakaryocytopoiesis and that the IL-11 levels we measured reflect a different aspect of its known pleotrophic properties (26).

In our neonates IL-11 was most commonly detectable during sepsis and NEC. Both these conditions are characterized by a local and systemic inflammatory response mediated by the major proinflammatory cytokines, tumor necrosis factor- $\alpha$, IL-1 $\beta$, and IL-6 (27-31). IL-11 is known to play a role in the endogenous pro- and counter-inflammatory response downregulating the proinflammatory response by modifying the release of the above cytokines (32). This action underlies the development of IL-11 as a therapeutic agent in conditions characterized by chronic inflammation [e.g. rheumatoid arthritis and Crohn's disease; $(26,33)]$. The elevated IL-11 levels we found in neonates with sepsis and NEC may therefore reflect their endogenous counter-inflammatory response rather than their megakaryocytopoietic or thrombopoietic status. This may also explain the elevated plasma IL-11 we found at birth in the three healthy neonates with a history of PROM and chorioamnionitis. Clearly further studies of IL-11 in healthy and sick or thrombocytopenic neonates will be required to define the significance of detectable IL-11 levels in these patients. To this end, we are currently conducting further studies to define the time course of changes in plasma IL-11 levels in neonatal patients with sepsis and NEC with or without thrombocytopenia to more specifically assess whether IL-11 is 1 ) involved in the regulation of platelet production in neonates; 2) an important endogenous counter-inflammatory cytokine in neonates; and 3) a potential therapy either as a stimulator of megakaryocytopoiesis and platelet production or as an immune modulator in sick neonatal patients.

In summary, the data presented in this paper show that healthy neonates with normal platelet counts do not have detectable levels of plasma IL-11. In contrast, many preterm neonates with proven sepsis or NEC have elevated plasma levels of IL-11. However, as there was no correlation between plasma IL-11 and platelet count, the significance of these findings are unclear. Data from our group, however, indicate that neonatal megakaryocyte precursor and progenitor cells do proliferate in vitro in response to IL-11 (20, McCloy, Roberts, Murray, unpublished data). Therefore, further work is indicated to delineate the role of IL-11 in neonatal platelet production in view of both the therapeutic availability of rhIL-11 and the high incidence of thrombocytopenia necessitating platelet transfusion in sick neonates. Finally, the observation that elevated IL-11 is most consistently seen during sepsis and NEC also deserves further evaluation in view of the potential role of IL-11 as an important counter-inflammatory cytokine in these potentially devastating inflammation-mediated conditions in neonates.

\section{REFERENCES}

1. Castle V, Andrew M, Kelton J, Giron D, Johnson M, Carter C 1986 Frequency and mechanism of neonatal thrombocytopenia. J Pediatr 108:749-755

2. Murray NA, Roberts IAG 1996 Circulating megakaryocytes and their progenitors in early thrombocytopenia in preterm neonates. Pediatr Res 40:112-119

3. Metha P, Rohitkumar V, Neumann L Karpatkin M 1980 Thrombocytopenia in the high risk infant. J Pediatr 97:791-794

4. Amato M, Fauchere JC, Herman Jr U 1988 Coagulation abnormalities in low birth weight infants with peri-intraventricular hemorrhage. Neuropediatrics 19:154-157

5. Andrew M, Castle V, Saigal S, Carter C, Kelton JG 1987 Clinical impact of neonatal thrombocytopenia. J Pediatr 110:457-464

6. Van De Bor M, Briet E, Van Bel F, Ruys JH 1986 Hemostasis and periventricularintraventricular hemorrhage of the newborn. Am J Dis Child 140:1131-1134

7. Setzer ES, Webb IB, Wassenaar JW, Reeder JD, Mehta PS, Eitzman DV 1982 Platelet dysfunction and coagulopathy in intraventricular hemorrhage in the premature infant. J Pediatr 100:599-605

8. Strauss RG, Levy GJ, Sotelo-Avila C, Albanese MA, Hume H, Schloz L, Blazina J, Werner A, Barrasso C, Blanchette V, Warkentin PI, Pepkowitz S, Mause AM, Hines D 1993 National survey of neonatal transfusion practices: II. Blood component therapy. Pediatrics 91:530-536 
9. Murray NA, Howarth LJ, McCloy MP, Letsky EA, Roberts IAG 2002 Platelet transfusion in the management of severe thrombocytopenia in neonatal intensive care unit (NICU) patients. Transfus Med 12:35-41

10. Andrew M, Castle V, Saigal S, Carter C, Kelton JG 1993 A randomized, controlled trial of platelet transfusions in thrombocytopenic premature infants. J Pediatr 123:285-291

11. Williamson LM 2000 Leucocyte depletion of the blood supply-how will patients benefit? Br J Haematol 110:256-272

12. Roberts IA, Murray NA 2001 Neonatal thrombocytopenia: new insights into pathogenesis and implications for clinical management. Curr Opin Pediatr 13:16-21

13. Sola MC, Calhoun DA, Hutson AD, Christensen RD 1999 Plasma thrombopoietin concentrations in thrombocytopenic and non-thrombocytopenic patients in a neonatal intensive care unit. Br J Haematol 104:90-92

14. Eaton DL, de Sauvage FJ 1997 Thrombopoietin: the primary regulator of megakaryocytopoiesis and thrombopoiesis. Exp Hematol 25:1-7

15. Watts TL, Murray NA, Roberts IAG 1999 Thrombopoietin has a primary role in the regulation of platelet production in preterm neonates. Pediatr Res 46:28-32

16. Albert TSE, Meng G, Simms P, Cohen RL, Phibbs RH 2000 Thrombopoietin in the thrombocytopenic term and preterm newborn. Pediatrics 105:1286-1291

17. Tepler I, Elias L, Smith JW, Hussein M, Rosen G, Chang AY, Moore JO, Gordon MS, Kuca B, Beach KJ, Loewy JW, Garnick MB, Kaye JA 1996 A randomized placebocontrolled trial of recombinant human interleukin-11 in cancer patients with severe thrombocytopenia due to chemotherapy. Blood 87:3607-3614

18. Isaacs C, Robert NJ, Bailey FA, Schuster MW, Overmoyer B, Graham M, Cai B, Beach KJ, Loewy JW, Kaye JA 1997 Randomized placebo-controlled study of recombinant human interleukin-11 to prevent chemotherapy-induced thrombocytopenia in patients with breast cancer receiving dose-intensive cyclophosphamide and doxorubicin. J Clin Oncol 15:3368-3377

19. Cairo MS, Kirov II, Goldman S, Davenport G, van de Ven C, Reaman G, Laver J, Kreissman S, Berg S, Blazar B 1998 Recombinant human interleukin-11 enhances hematologic recovery following ICE chemotherapy in children with solid tumors or lymphoma: analysis of hematopoietic responses, cytokine induction, pharmacokinetics and stem cell mobilization. Proc ASCO 17:538a(abstr)

20. McCloy MP, Howarth LJ, Watts TL, Murray NA, Roberts IAG 2001 The role of IL-11 in neonatal thrombocytopenia. Blood 96:564a(abstr)

21. Cipriani CA, Wagnon MA, Kuehl TJ 2001 Measurement of human interleukin-11 in neonates. Pediatr Res 49:395A(abstr)

22. Cremer M, Schulze H, Linthorst G, Folman CC, Wehnert S, Strauss G, von dem Borne AE, Welte K, Ballmaier M 1999 Serum levels of thrombopoietin, IL-11, and IL-6 in pediatric thrombocytopenias. Ann Hematol 78:401-407
23. Chang M, Suen Y, Meng G, Buzby JS, Bussel J, Shen V, van de Ven C, Cairo MS 1996 Differential mechanisms in the regulation of endogenous levels of thrombopoietin and interleukin-11 during thrombocytopenia: insight into the regulation of platelet production. Blood 88:3354-3362

24. Tahara T, Usuki K, Sato H, Ohashi H, Morita H, Tsumura H, Matsumoto A 1996 A sensitive sandwich ELISA for measuring thrombopoietin in human serum: serum thrombopoietin levels in healthy volunteers and in patients with haemopoietic disorders. Br J Haematol 93:783-788

25. Emmons RV, Reid DM, Cohen RL, Meng G, Young NS, Dunbar CE, Shulman NR 1996 Human thrombopoietin levels are high when thrombocytopenia is due to megakaryocyte deficiency and low when due to increased platelet destruction. Blood $87: 4068-4071$

26. Schwertschlag US, Trepicchio WL, Dykstra KH, Keith JC, Turner KJ, Dorner AJ 1999 Hemopoietic, immunomodulatory and epithelial effects of interleukin-11. Leukemia 13:1307-1315

27. Edelson MB, Bagwell CE, Rozycki HJ 1999 Circulating pro- and counterinflammatory cytokine levels and severity in necrotizing enterocolitis. Pediatrics 103:766-771

28. Morecroft JA. Spitz L, Hamilton PA, Holmes SJ 1994 Plasma interleukin-6 and tumour necrosis factor levels as predictors of disease severity and outcome in necrotizing enterocolitis. J Pediatr Surg 29:798-800

29. Harris MC, Costarino Jr AT, Sullivan JS, Dulkerian S, McCawley L, Corcoran L, Butler S, Kilpatrick L 1994 Cytokine elevations in critically ill infants with sepsis and necrotizing enterocolitis. J Pediatr 124:105-111

30. Silveira RC, Procianoy RS 1999 Evaluation of interleukin-6, tumour necrosis factoralpha and interleukin-1beta for early diagnosis of neonatal sepsis. Acta Paediatr 88:647-650

31. Kuster H, Weiss M, Willeitner AE, Detlefsen S, Jeremias I, Zbojan J, Geiger R, Lipowsky G, Simbruner G 1998 Interleukin-1 receptor antagonist and interleukin-6 for early diagnosis of neonatal sepsis 2 days before clinical manifestation. Lancet 352:1271-1277

32. Peterson RL, Wang L, Albert L, Keith Jr JC, Doner AJ 1998 Molecular effects of recombinant human interleukin-11 in the HLA-B27 rat model of inflammatory bowel disease. Lab Invest 78:1503-1512

33. Sands B, Bank S, Sninsky C, Robinson M, Katz S, Singleton J, Miner P, Safdi M, Galandiuk S, Hanauer S, Varilek G, Buchman A, Rogers V, Salzberg B, Cai B, Rogge H, Schwertschlag U 1999 Safety and activity evaluation of rhIL-11 in subjects with active Crohn's disease. Gastroenterology 117:1-8 